\title{
Closed timelike curves and geodesics of Godel-type* metrics
}

Sar1o“

\author{
Reinaldo J Gleiser ${ }^{1}$, Metin Gurses ${ }^{* 2}$, Atalay Karasu ${ }^{3}$ and Ozg“ ur \\ glu $^{-3}$ \\ ${ }^{1}$ Facultad de Matematica, Astronom' '1a y F'1sica, Universidad Nacional de Cordoba, \\ Ciudad Universitaria, (5000), Cordoba, Argentina \\ ${ }^{2}$ Department of Mathematics, Faculty of Sciences, Bilkent University, 06800, Ankara, Turkey \\ ${ }^{3}$ Department of Physics, Faculty of Arts and Sciences, Middle East Technical University, 06531, \\ Ankara, Turkey \\ E-mail: gleiser@fis.uncor.edu, gurses@fen.bilkent.edu.tr, karasu@metu.edu.tr and \\ sarioglu@metu.edu.tr
}

Received 7 December 2005, in final form 29 January 2006

Published 17 March 2006

Online at stacks.iop.org/CQG/23/2653

\begin{abstract}
It is shown explicitly that when the characteristic vector field that defines a Godel-type metric is also a Killing vector, there always exist closed timelike* or null curves in spacetimes described by such a metric. For these geometries, the geodesic curves are also shown to be characterized by a lower-dimensional Lorentz force equation for a charged point particle in the relevant Riemannian background. Moreover, two explicit examples are given for which timelike and null geodesics can never be closed.
\end{abstract}

PACS numbers: 04.20.Jb, 04.40.Nr, 04.50.+h, 04.65.+e

\section{Introduction}

ThecelebratedGodelmetric[“ 1] $\quad$ solvestheEinsteinfieldequationswithahomogeneousperfect fluid source and has a $\mathrm{G}_{5}$ maximal symmetry [2]. The Godel spacetime admits closed timelike" and closed null curves but contains no closed timelike or closed null geodesics [3]. Moreover, the Godel universe is geodesically complete and it has neither a singularity nor a horizon."

In its original form, the Godel metric reads"

$$
\mathrm{d}^{S^{2}}=-\left(\mathrm{d} x^{0}\right)^{2}+\left(\mathrm{d} x^{1}\right)^{2}-\frac{1}{2} e^{2 x^{1}}\left(\mathrm{~d} x^{2}\right)^{2}+\left(\mathrm{d} x^{3}\right)^{2}-2 e^{x^{1}} \mathrm{~d} x^{0} \mathrm{~d} x^{2} .
$$

(In fact Godel's original metric has an overall constant factor" $\quad \mathrm{a}^{2}$ multiplying the line element. We have taken $\mathrm{a}=1$ here.) A simple rearrangement of the terms yields that (1) can also be written as

$$
\mathrm{d}^{s^{2}}=\left(\mathrm{d} x^{1}\right)^{2}+\frac{1}{2} e^{2 x^{1}}\left(\mathrm{~d} x^{2}\right)^{2}+\left(\mathrm{d} x^{3}\right)^{2}-\left(\mathrm{d} x^{0}+e^{x^{1}} \mathrm{~d} x^{2}\right)^{2} .
$$

0264-9381/06/072653+11\$30.00 (c) 2006 IOP Publishing Ltd Printed in the UK

This form (2) suggests that the Godel metric can be thought of as cast in the form"

$$
\mathrm{g}_{\mu v}=\mathrm{h}_{\mu v}-\mathrm{u} \mu \mathrm{u} v,
$$


where the 'background' $\mathrm{h}_{\mu v}$ is a non-flat 3-metric and $\mathrm{u}_{\mu}=\delta_{\mu}{ }^{0}+\mathrm{e}^{\mathrm{x}_{1}} \delta_{\mu}{ }^{2}$ is a timelike unit vector: $\mathrm{g}^{\mu v} \mathbf{u}_{\mu} \mathbf{u}_{v}=-1$. Note, however, that (2) is not the only way of rearranging the terms in (1). One could as well rewrite it as

$$
\mathrm{d}^{s^{2}}=\left(\mathrm{d} x^{0}\right)^{2}+\left(\mathrm{d} x^{1}\right)^{2}+\left(\mathrm{d} x^{3}\right)^{2}-\left(\sqrt{ } 2 \mathrm{~d} x^{0}+\frac{1}{\sqrt{2}} e^{x^{1}} \mathrm{~d} x^{2}\right)^{2},
$$

which again can be viewed in the form of (3); however, this time the 'background' $h_{\mu v}$ $=\mathrm{V} \operatorname{diag}(1,1,0 \mathrm{~V}, 1)$ describes an obviously flat three-dimensional spacetime and the new 1

$$
\mathrm{u}_{\mu}=2 \delta_{\mu}{ }^{0}+(1 / \overline{2}) \mathrm{e}^{\mathrm{x}} \delta_{\mu}{ }^{2} \text { is again a timelike unit vector in this new form. }
$$

Inspired by these observations, a class of $D$-dimensional metrics were introduced, which were christened Godel-type metrics", and used for producing new solutions to various gravitational theories in diverse dimensions [4,5]. Stated briefly, these metrics are of the form (3), where the background $h_{\mu v}$ is the metric of an Einstein space of a (D - 1)-dimensional Riemannian geometry in the most general case and $\mathrm{u}^{\mu}$ is a timelike unit vector. A further assumption is that both $\mathrm{h}_{\mu v}$ and $\mathrm{u}_{\mu}$ are independent of the fixed special coordinate $\mathrm{x}^{\mathrm{k}}$ with

$0 \leqslant k \leqslant D-1$ and, moreover, that $\mathrm{h}_{\mathrm{k} \mu}=0$. A detailed analysis was given corresponding to the two distinct cases $u_{\mathrm{k}}=$ constant and $u_{k} \neq$ constant, where $u^{\mu}=-\delta_{k}^{\mu} / u_{k}$, in [4] and

[5], respectively. It was shown that Godel-type metrics with" $u_{k}=$ constant can be used in constructing solutions to the Einstein-Maxwell field equations with a dust distribution in $D$ dimensions and in that case the only essential field equation turns out to be the source-free 'Maxwell's equation' in the relevant background [4]. One also finds that $\mathrm{u}^{\mu}$ is tangent to a timelike geodesic curve that is also a timelike Killing vector [4]. Moreover, it was established in [5] that when $u_{k} \neq$ constant, the conformally transformed Godel-type metrics can be used" in solving a rather general class of Einstein-Maxwell-dilaton-3-form field theories in $D \geqslant 6$ dimensions and that, in this case, all field equations can be reduced to a simple 'Maxwell equation' in the corresponding (D -1)-dimensional Riemannian background. However, with $u_{k} \neq$ constant, $\mathrm{u}^{\mu}$ is no longer a Killing vector field unlike the constant case [5]. In these works, it was also shown that Godel-type metrics can be used in obtaining exact solutions to" various supergravity theories, in which case $\mathrm{u}_{\mathrm{k}}$ may be considered as related to a dilaton field $\varphi$ via the relationship $\varphi=\ln \left|u_{k}\right|$. (See $[4,5]$ for the details.)

The existence of the closed timelike and closed null curves in the Godel spacetime can be" best inferred by transforming (1) to the cylindrical coordinates in which case it reads

$$
d s^{2}=-d t^{2}+d r^{2}+d z^{2}-\sinh ^{2} r\left(\sinh ^{2} r-1\right) d \phi^{2}+2 \sqrt{2} \sinh ^{2} r d \phi d t .
$$

It readily follows that the curve $\mathrm{C}=\left\{(\mathrm{t}, \mathrm{r}, \phi, \mathrm{z}) \mid \mathrm{t}=\mathrm{t}_{0}, \mathrm{r}=\mathrm{r}_{0}, \mathrm{z}=\mathrm{z}_{0} \mathrm{~V}, \phi \in[0,2 \pi]\right\}$, where $\mathrm{t}_{0}, \mathrm{r}_{0}$ and $\mathrm{z}_{0}$ are constants, is a closed timelike curve for $\mathrm{r}_{0}>\ln (1+2)$ and a closed null

curve for $r_{0}=\ln (1+\sqrt{ } 2)$.

There have been attempts to remove such closed timelike curves from spacetimes described by Godel-type metrics by introducing observer-dependent holographic screens ["

$6,7]$ in the context of supergravity theories. Other 'remedies' that have been put forward to remove such curves involve the addition of scalar fields [8], more specifically dilaton and axion fields [9], to change the matter content, and to consider theories that involve higher order curvature terms in their local gravity action $[10,11]$. The discussion of the closed timelike curves in these works, e.g. [8,9], seems to be restricted to an investigation of the 
curves parametrized as the curve $C$ above, and thus solely on the general behaviour of the metric component $\mathrm{g}_{\phi \phi}$ 
since they use a general metric ansatz

$$
d s^{2}=-[d t+C(r) d \phi]^{2}+D^{2}(r) d \phi^{2}+d r^{2}+d z^{2}
$$

that respects the cylindrical symmetry and generalizes the Godel metric ("5).

The discussion regarding the existence of closed timelike or null curves and the behaviour of geodesics in Godel-type metrics with" $\mathrm{u}_{\mathrm{k}}=$ constant had to be kept concise in [4]; thus only curves parametrized as the curve $C$ above were considered. However, it is obvious that there can be other classes of curves that can be both closed and timelike (or null). The aim of the present work is to provide a much more detailed analysis of these special curves in geometries described by such metrics. Specifically, it is going to be proved explicitly that the non-flat spacetimes described by Godel-type metrics with both flat and non-flat backgrounds always" have closed timelike or null curves. As a separate discussion, it will also be shown that the geodesics of Godel-type metrics with constant" $u_{k}$ are characterized by the (D-1)-dimensional LorentzforceequationforachargedpointparticleformulatedinthecorrespondingRiemannian background.

\section{Closed timelike curves in Godel-type metrics with flat backgrounds and constant"}

$u_{k}$

In this section, we will assume without loss of generality that the fixed special coordinate $\mathrm{x}^{\mathrm{k}}$ equals $\mathrm{x}^{0} \equiv \mathrm{t}$, the background $\mathrm{h}_{\mu \nu}$ describes a flat Riemannian geometry, $\mathrm{h}_{0 \mu}=0$ and $\mathrm{u}_{0}=1$. We will also take $\mathrm{D}=4$ for simplicity but what follows can easily be generalized to higher dimensions.

In subsection 2.4 of [4], spacetimes with the line element

$$
d s^{2}=d \rho^{2}+\rho^{2} d \varphi^{2}+d z^{2}-(d t+s(\rho, \varphi) d z)^{2}
$$

were considered. It was found that this metric (6) is a solution of the charged dust field equations in four dimensions provided $s(\rho, \varphi)$ is a harmonic function in two dimensions. Then the simplest possible choice, namely $\mathrm{s}=$ constant and a rather plain and specific curve of the form $\mathrm{C}^{-}=\left\{(\mathrm{t}, \rho, \varphi, \mathrm{z}) \mid \mathrm{t}=\mathrm{t}_{0}, \rho=\rho_{0}, \varphi=\varphi_{0}, \mathrm{z} \in[0,2 \pi)\right\}$, where $\mathrm{t}_{0}, \rho_{0}$ and $\varphi_{0}$ are constants, was considered. Then it was shown that the tangent vector ${ }^{-} \mathrm{v}^{\mu}=(\partial / \partial \mathrm{z})^{\mu}$ is timelike if $\left(\mathrm{s}\left(\rho_{0}, \varphi_{0}\right)\right)^{2}>$ 1. However, it was argued that one could exclude these closed timelike curves by considering the $\mathrm{z}$ coordinate to be in the universal covering of this patch, namely by taking $\mathrm{z}$ to be on the real line R. Finally, this subsection was closed with the statement: we thus conclude that the solutions we present correspond to spacetimes that contain both closed timelike and null curves and that contain neither of these depending on how one solves (7). (Here (7) refers to the flat three-dimensional Euclidean source-free 'Maxwell's equation' $\partial_{\mathrm{i}} \mathrm{f}_{\mathrm{ij}}=0$, where $\mathrm{i}, \mathrm{j}$ indices range from 1 to 3 and $f_{i j} \equiv \partial_{i} u_{j}-\partial_{j} u_{i}$.)

It readily follows that the 'Maxwell equation' $\partial_{\mathrm{i}} \mathrm{f}_{\mathrm{ij}}=0$ is equivalent to the Laplace equation for the (nontrivial) function $s(\rho, \varphi)$ in two dimensions. In [4], it was implicitly assumed that there were nontrivial harmonic functions suitable for the discussion carried on and the 
conclusion reached in subsection 2.4. However, we want to show in what follows that the previous discussion does not necessarily exclude the possibility of other closed timelike curves.

Let us, first of all, consider any smooth timelike curve in the spacetime described by (6) with constant $\rho$ and $\varphi$, so that $\left(\mathrm{t}(\eta), \rho_{0}, \varphi_{0}, z(\eta)\right)$ is a parametrization of with a normalized tangent vector

$$
\begin{array}{r}
\left(\frac{\mathrm{d} z}{\mathrm{~d} \eta}\right)^{2}-\left(\frac{\mathrm{d} t}{\mathrm{~d} \eta}+s_{0} \frac{\mathrm{d} z}{\mathrm{~d} \eta}\right)^{2}=-1, \\
, \begin{array}{l}
\text { where } \mathrm{s}_{0} \equiv \mathrm{s}\left(\rho_{0}, \varphi_{0}\right)=\text { constant. } \\
\text { solved for } \mathrm{dt} / \mathrm{d} \eta \text { and formally integrated on } \eta \text { to get }
\end{array} \\
t(\eta)=t(0)-s_{0}[z(\eta)-z(0)]+\epsilon \int_{0}^{\eta}\left[1+\left(\frac{\mathrm{d} z}{\mathrm{~d} \eta^{\prime}}\right)^{2}\right]^{1 / 2} \mathrm{~d} \eta^{\prime}, \quad \text { where } \epsilon= \pm 1 .
\end{array}
$$

Since the integral in (8) is an increasing function of $\eta$, this shows that there are no smooth timelike curves along which both $t$ and $\mathrm{z}$ simultaneously recover their initial values and, therefore, there are no closed timelike curves of this type for the metric (6). However, for $\eta \gg 1$, one may have timelike curves where both $t$ and z perform rather large excursions, but then come back to values that are close to their initial values.

This brings to mind the question whether it could be possible to obtain closed timelike curves if one allows also a variation in $\rho$ and/or $\varphi$. As an example, consistent with the assumption of being harmonic, consider a neighbourhood of a point $\left(\rho_{0}, \varphi_{0}\right)$, and assume that, for fixed $\varphi$, the function $s(\rho, \varphi)$ takes the form

$$
s(\rho, \varphi)=s_{0}+s_{1}\left(\rho-\rho_{0}\right),
$$

where $\mathrm{s}_{0}$ and $\mathrm{s}_{1}$ are constants. Then, one can check that the curve

$$
\begin{aligned}
& \rho(\eta)=\rho_{0}+a \cos \eta, \quad \phi=\phi_{0}, \quad z(\eta)=a \sin \eta, \\
& t(\eta)=-a\left[s_{0}+\frac{a s_{1}}{2} \cos \eta\right] \sin \eta
\end{aligned}
$$

is a smooth timelike curve with a normalized tangent vector, provided that $a$ is a solution of

$$
s_{1}^{2} a^{4} \quad 4 a^{2} \quad 4=0
$$

A suitable solution of (11) is

$$
a=\frac{\sqrt{2+2 \sqrt{1+s_{1}^{2}}}}{s_{1}}, \quad s_{1} \neq 0,
$$

which is well defined for all values of $s_{1}$. Since the coordinates $(t, \rho, z)$ are all periodic in $\eta$, one concludes that (10) plus (12) represents a smooth closed timelike curve for the metric (6).

This was only a counter example for the assertion that (6) is an example of a spacetime without closed timelike curves. It follows from this analysis that in order to determine the absence (or the presence) of closed timelike curves, one would need to consider in each case the explicit form of the function $\mathrm{s}(\rho, \varphi)$. As for another, more general, example, consider

$$
\mathrm{s}_{\mathrm{n}}(\rho, \varphi)=\gamma+\rho^{\mathrm{n}}(\alpha \cos n \varphi+\beta \operatorname{sinn} \varphi), \quad \mathrm{n} \in Z^{+},
$$


where $\alpha, \beta$ and $\gamma$ are real constants. (Obviously this function is related to the real and imaginary parts of the complex analytic function $g(\zeta)=\zeta^{\mathrm{n}}, \mathrm{n} \in \mathrm{Z}^{+}$.) It readily follows that the nontrivial components of the Maxwell (matter) field

$$
f_{p z}=n \rho^{n-1}(\alpha \cos n \varphi+\beta \sin n \varphi), \quad f_{\varphi z}=n \rho^{n}(-\alpha \sin n \varphi+\beta \cos n \varphi)
$$

now depend on the constants $\alpha$ and $\beta$, and do not vanish for all $(\rho, \varphi)$ unless $\alpha, \beta \rightarrow 0$.

Letusnowtakethecurve $\mathrm{e}^{\sim} \quad$ (similartothecurve above)definedby $\left(\mathrm{t}(\eta), \rho(\eta), \varphi_{0}, \mathrm{z}(\eta)\right)$, whereonecanassumethattheparameter $\eta$ takesvaluesin $[0,2 \pi]$ withoutanylossofgenerality, $\rho(\eta)$ $=\rho_{0}+a \cos \eta, z(\eta)=a \sin \eta,(14)$

and $\varphi_{0}, \rho_{0}$ and $a$ are real constants as before. Using the line element (6) with $\mathrm{s}_{\mathrm{n}}(\rho, \varphi)$ given in (13) and solving the constraint equation that normalizes the tangent vector of this curve to unity, one finds that $\mathrm{d}$

${ }^{t}=\epsilon \sqrt{1+a^{2}} \quad-\quad-\quad-\mathrm{as}_{\mathrm{n}}\left(\rho, \varphi_{0}\right) \cos \eta, \quad \epsilon= \pm 1 \quad$ where, again,

$\mathrm{d} \eta$

and

$\mathrm{s}_{\mathrm{n}}\left(\rho, \varphi_{0}\right)=\gamma+\sigma_{\mathrm{n}}\left(\rho_{0}+\mathrm{a} \cos \eta\right)^{\mathrm{n}} \quad$ with $\quad \sigma_{\mathrm{n}} \equiv \alpha \operatorname{cosn} \varphi_{0}+\beta \operatorname{sinn} \varphi_{0}$.

When (15) is integrated for $t$, the outcome can be put in a form

$t(\eta)=f_{n}(\eta)+\left(A_{n} \sigma_{n}+\epsilon \sqrt{1+a^{2}}\right) \eta+t_{0}, \quad t_{0}=$ integration constant,

where $f_{n}(\eta$ ) is a periodic function of $\eta$ that consists of a linear sum of sinm $\eta$ terms (with $m=$ $0,1, \ldots, n+1)$ and $A_{n}$ is another constant term, both of which depend naturally on the choice of the constant $n \in Z^{+}$. (Using the notation introduced thus far, one gets

$A_{n}= \begin{cases}-a^{2} / 2, & n=1 \\ -a^{2} \rho_{0}, & n=2,\end{cases}$

$f_{n}(\eta)= \begin{cases}-a\left(\gamma+\sigma_{1} \rho_{0}\right) \sin \eta-\left(a^{2} \sigma_{1} / 4\right) \sin 2 \eta, & n=1 \\ -\left(a\left(\gamma+\sigma_{2} \rho_{0}^{2}\right)+3 a^{3} \sigma_{2} / 4\right) \sin \eta-\left(a^{2} \rho_{0} \sigma_{2} / 2\right) \sin 2 \eta-\left(a^{3} \sigma_{2} / 12\right) \sin 3 \eta, & n=2,\end{cases}$ for the simplest cases $n=1$ and $n=2$.) Similar to how the $t(\eta)$ part of the curve was made a periodic function of $\eta$ above, one can set the coefficient of the $\eta$ term on the right-hand side of (17) to zero by suitably choosing the constant $A_{n}$, provided the constant $\sigma_{n}$ does not tend to zero or that $\mathrm{s}_{\mathrm{n}}(\rho, \varphi)$ does not go to a constant. One again concludes that $~$, as described above, represents a smooth closed timelike curve for the metric (6).

A natural question to address then is whether closed timelike or null curves exist for all harmonic functions. The answer one finds is as follows.

Consider the most general curve $C$ defined by $(t(\eta), \rho(\eta), \varphi(\eta), z(\eta))$, where the arclength parameter $\eta$ takes values in the simple (generic) interval $[0,2 \pi]$. Normalizing the tangent vector of this curve $C$ to unity in the geometry described by (6), one finds 


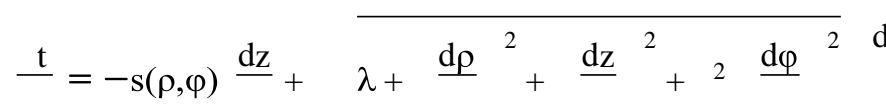

$$
\begin{aligned}
& \mathrm{d} \eta \quad \mathrm{d} \eta \quad \mathrm{d} \eta \quad \mathrm{d} \eta \quad \mathrm{d} \eta
\end{aligned}
$$

where $\lambda=0$ for null and $\lambda=1$ for timelike curves. Now let the parametrizations of $\rho, \varphi$ and $z$ be all periodic functions in $\eta$. Then the terms in the square root in (18) can be expanded in a Fourier series in the interval $[0,2 \pi]$ and it is clear that this Fourier series expansion has a nonnegative constant term in it which looks like ${ }^{4}$

$$
B=\frac{1}{2 \pi} \int_{0}^{2 \pi} \sqrt{\lambda+\left(\frac{\mathrm{d} \rho}{\mathrm{d} \eta}\right)^{2}+\left(\frac{\mathrm{d} z}{\mathrm{~d} \eta}\right)^{2}+\rho^{2}\left(\frac{\mathrm{d} \phi}{\mathrm{d} \eta}\right)^{2}} \mathrm{~d} \eta>0 .
$$

As it stands, $\operatorname{since} B \neq 0, t(\eta)$ naturally picks up a non-periodic piece $B \eta$ from the second term on the right-hand side of (18) and in order to have no closed timelike (or null) curves, it must be that

$$
\int_{0}^{2 \pi} s(\rho(\eta), \phi(\eta)) \frac{\mathrm{d} z}{\mathrm{~d} \eta} \mathrm{d} \eta=
$$

for all arbitrary periodic functions $z(\eta)$. However, since $\rho(\eta)$ and $\varphi(\eta)$ are periodic functions of $\eta$, it readily follows that $s(\rho(\eta), \varphi(\eta))$ is also periodic in $\eta$. (In fact, it is enough to demand that $s(\rho, \varphi)$ is a continuous function, rather than a harmonic function, of its arguments for the discussion that follows. We would like to stress that the condition for being harmonic

4 Strictly speaking, $B$ is positive definite for $\lambda=1$ and non-negative for $\lambda=0$. When $\lambda=0, \mathrm{~B}=0$ iff $\rho, \varphi$ and $\mathrm{z}$ are all constants, i.e. $s(\rho, \varphi)=$ constant which we have already discussed. Hence, for the discussion that follows we assume that $\mathrm{B}>0$.

followed from the Maxwell equation (7) of [4].) In this case, one can expand $s(\rho(\eta), \varphi(\eta))$ in a Fourier series in $\eta$ as

$$
s(\rho(\eta), \phi(\eta))=a_{0}+g(\eta)=a_{0}+\sum_{p=1}^{\infty}\left(a_{p} \cos p \eta+b_{p} \sin p \eta\right),
$$

where $a_{0}, a_{k}$ and $b_{k}$ are the Fourier coefficients in the usual manner. Now (19) implies that

$$
\int_{0}^{2 \pi} g(\eta) \frac{\mathrm{d} z}{\mathrm{~d} \eta} \mathrm{d} \eta=0
$$

If one chooses the periodic function $\mathrm{z}(\eta)$ so that $\mathrm{dz} / \mathrm{d} \eta=\mathrm{g}(\eta)$, then

$$
\int_{0}^{2 \pi}(g(\eta))^{2} \mathrm{~d} \eta=0
$$

which is possible only if $g(\eta)=0$, and hence $s(\rho, \varphi)=$ constant. Therefore, unless $s(\rho, \varphi)=$ constant, one can always cancel out the contribution of the $B$ term above and find a closed timelike or null curve in the spacetime described by (3).

We thus see that the conclusion reached at the end of subsection 2.4 of [4] needs to be corrected as follows: there exists no nontrivial (non-constant) harmonic function $s(\rho, \varphi)$ such that the spacetime described by (6) has no closed timelike or null curves; i.e. one can always 
find a closed timelike or null curve in the geometry of (6) given an arbitrary harmonic function $\mathrm{s}(\rho, \varphi)$.

\section{Closed timelike curves in Godel-type metrics with non-flat backgrounds and" constant $u_{k}$}

Let us now see how the results of section 2 can also be applied to Godel-type metrics with" non-flat backgrounds but with constant $\mathrm{u}_{\mathrm{k}}$. Let us once again assume without loss of generality that the fixed special coordinate $\mathrm{x}^{\mathrm{k}}$ equals $\mathrm{x}^{0} \equiv \mathrm{t}$, the background $\mathrm{h}_{\mu v}$ is the metric of an Einstein space of a (D-1)-dimensional (non-flat) Riemannian geometry, $\mathrm{h}_{0 \mu}=0$ and $\mathrm{u}_{0}=1$. To keep the discussion simple, let us take $\mathrm{D}=4$ as before; however, the following can be generalized to higher dimensions without any difficulty.

In [4], it was found that the Godel-type metric"

$$
\mathrm{d}^{s^{2}}=h_{i j}\left(x^{\ell}\right) \mathrm{d} x^{i} \mathrm{~d} x^{j}-\left(\mathrm{d} t+u_{i}\left(x^{\ell}\right) \mathrm{d} x^{i}\right)^{2},
$$

where indices i,j, range from 1 to 3 , is a solution to the Einstein-Maxwell field equations with a dust distribution provided that the three-dimensional source-free 'Maxwell equation'

$$
\partial_{\alpha}\left(\bar{h}^{\alpha \mu} \bar{h}^{\beta \nu} \sqrt{|h|} f_{\mu v}\right)=0,
$$

where $\mathrm{f}_{\mu v} \equiv \partial_{\mu} \mathrm{u}_{v}-\partial_{v} \mathrm{u}_{\mu}$ as before and $\mathrm{h}^{-\mu v}$ is the three-dimensional inverse of $\mathrm{h}_{\mu v}$, is satisfied.

Starting with a specific background $\mathrm{h}_{\mu v}$, one should be able, in principle, to solve

(21) and use the solution vector $u_{i}$ to write down (20) explicitly. It should be kept in mind that $h_{i j}=h_{i j}\left(x^{\ell}\right)$ by assumption and, solving $(21), u_{i}=u_{i}\left(x^{\ell}\right)$ in the most general case.

Now let us consider a general curve $C$ defined by $\left(\mathrm{t}(\eta), \mathrm{x}^{\mathrm{i}}(\eta)\right)$ parametrized with the arclength parameter $\eta \in[0,2 \pi]$ as in section 2 . Normalizing the tangent vector of $C$ to unity by using (20), one obtains

$$
\underline{t}=-u(x) \frac{d x^{i}}{\lambda+h(x) \frac{d x^{i}}{d x^{j}}} d
$$

$$
\mathrm{d} \eta \quad \mathrm{d} \eta \quad \mathrm{d} \eta \mathrm{d} \eta
$$

where $\epsilon= \pm 1, \lambda=0$ for null and $\lambda=1$ for timelike curves. Now let the parametrization of each $x^{i}$ be a periodic function in $\eta$. One can then follow similar steps to those described in section 2 and use Fourier series expansion of the terms on the right-hand side of (22). One finds that the Fourier series expansion has a non-vanishing constant term of the form ${ }^{5}$

$$
\beta=\frac{1}{2 \pi} \int_{0}^{2 \pi} \sqrt{\lambda+h_{i j}\left(x^{\ell}\right) \frac{\mathrm{d} x^{i}}{\mathrm{~d} \eta} \frac{\mathrm{d} x^{j}}{\mathrm{~d} \eta}} \mathrm{d} \eta>0,
$$

which contributes a term $\beta \eta$ to the integration of (22) for $t(\eta)$.

In order to have no closed timelike (or null) curves, one must have 


$$
\sum_{i=1}^{3} \int_{0}^{2 \pi} u_{i}\left(x^{\ell}\right) \frac{\mathrm{d} x^{i}}{\mathrm{~d} \eta} \mathrm{d} \eta=
$$

for all arbitrary periodic functions $\mathrm{x}^{\mathrm{i}}(\eta)$. However, each $u_{i}\left(x^{\ell}\right)$ is also periodic in $\eta$ since each $x^{\ell}(\eta)$ is. Moreover, even though their explicit form may not be available, the functions $u_{i}\left(x^{\ell}\right)$ are also continuous functions of $\eta$ since they satisfy a second order (nonlinear) partial differential equation (21). Thus these functions may also be expanded in a Fourier series as

$$
u_{i}\left(x^{\ell}\right)=a_{i 0}+g_{i}(\eta)=a_{i 0}+\sum_{p=1}^{\infty}\left(a_{i p} \cos p \eta+b_{i p} \sin p \eta\right)
$$

with $\mathrm{a}_{\mathrm{i} 0}, \mathrm{a}_{\mathrm{ip}}$ and $\mathrm{b}_{\mathrm{ip}}$ denoting the Fourier coefficients in the usual sense. Now (23) implies that

$$
\sum_{i=1}^{3} \int_{0}^{2 \pi} g_{i}(\eta) \frac{\mathrm{d} x^{i}}{\mathrm{~d} \eta} \mathrm{d} \eta=0
$$

If one chooses the periodic functions $x^{\mathrm{i}}(\eta)$ such that $\mathrm{dx} / \mathrm{d} \eta=\mathrm{g}_{\mathrm{i}}(\eta)$ for each $i$, then

$$
\sum_{i=1}^{3} \int_{0}^{2 \pi}\left(g_{i}(\eta)\right)^{2} \mathrm{~d} \eta=0
$$

which is possible only if $g_{i}(\eta)=0$ and hence $u_{i}\left(x^{\ell}\right)=$ constant for each $i$. However, one can then define a new coordinate $d \tau=d t+u_{i} d x^{i}$ and can immediately see that (20) has no closed timelike or null curves. Hence, unless each one of $u_{i}\left(x^{\ell}\right)$ is a constant, one can always find a closed timelike or null curve in the spacetime (20) by smartly choosing a parametrization that cancels out the contribution of the $\beta$ term in the $t(\eta)$ equation.

We thus see that the conclusion reached at the end of section 2 can be extended to include all Godel-type metrics of the form (*20). When each $u_{i}\left(x^{\ell}\right)=$ constant, the spacetime is obviously flat and there exist no closed timelike or null curves. In retrospect, we have proved that the spacetimes described by Godel-type metrics with both flat and non-flat backgrounds " always have closed timelike or null curves, provided that at least one of the $u_{i}\left(x^{\ell}\right) \neq$ constant.

\section{Geodesics of Godel-type metrics with constant" $u_{k}$}

Let us now examine the geodesics of Godel-type metrics. In [“4], it was shown that Godeltype " metrics with flat backgrounds and constant $u_{k}$ have their geodesics described by the Lorentz force equation for a charged particle in the corresponding (D - 1)-dimensional Riemannian background. Let us now look into how the geodesics of a Godel-type metric with a general" non-flat background (but still with constant $u_{k}$ ) behave. To simplify the discussion, let us

each5 Once again, $\beta$ is in fact positive definite for $\lambda=1$ and non-negative for $\lambda=0$. However, when $\lambda=0, \beta=0$ iff $\mathrm{xi}(\eta)$ is a constant, and in that case (20) obviously has no closed null curves since by assumption the background $\mathrm{h}_{\mu v}$ has positive definite signature. Hence, we assume that $\beta>0$ for what follows.

again keep the general assumptions listed at the beginning of section 3, but this time let us keep $D$ arbitrary. 
The inverse of the Godel-type metric (" 3) is (as already given in (2) of [4]) $\mathrm{g}_{\mu \nu}=\mathrm{h}^{-}{ }_{\mu \nu}+$

$$
\left(-1+h^{-}{ }_{\alpha \beta} \mathbf{U}_{\alpha} \mathrm{u}_{\beta}\right) \mathrm{u}_{\mu} \mathrm{U}_{v}+\mathrm{u}_{\mu}\left(\mathrm{h}^{-}{ }^{-} \alpha \mathrm{U}_{\alpha}\right)+\mathrm{uv}_{v}\left(\mathrm{~h}^{-}{ }_{\mu \alpha \mathrm{u} \alpha}\right),(24)
$$

with $\mathrm{h}^{-\mu v}$ denoting the (D-1)-dimensional inverse of $h_{\mu v}$, as before. The Christoffel symbols of (3) in this case were also given by (37) of [4] as

$$
\Gamma_{\alpha \beta}^{\mu}=\gamma_{\alpha \beta}^{\mu}+\frac{1}{2}\left(u_{\alpha} f_{\beta}^{\mu}+u_{\beta} f_{\alpha}^{\mu}\right)-\frac{1}{2} u^{\mu}\left(u_{\alpha \mid \beta}+u_{\beta \mid \alpha}\right)
$$

in terms of the Christoffel symbols $\gamma^{\mu}{ }_{\alpha \beta}$ of $h_{\mu v}$ and the 'Maxwell field' $f_{\mu \nu} \equiv \partial_{\mu} u_{v}-\partial_{v} \mathbf{u}_{\mu}$, as before. Here a vertical stroke denotes a covariant derivative with respect to $h_{\mu v}$ and it should be kept in mind that the indices on $\mathrm{f}$ and $u$ are raised and lowered by the metric $\mathrm{g}_{\mu v}$.

With all the ingredients already at hand, let us now consider a geodesic curve in the spacetime described by (3) which is parametrized as $\mathrm{x}^{\mu}(\tau)$. Using (25) and denoting derivative with respect to the affine parameter $\tau$ by a dot, the geodesic equation yields

$$
\ddot{x}^{\mu}+\gamma_{\alpha \beta}^{\mu} \dot{x}^{\alpha} \dot{x}^{\beta}+\left(u_{\alpha} \dot{x}^{\alpha}\right)\left(f_{\beta}^{\mu} \dot{x}^{\beta}\right)-u^{\mu}\left(u_{\alpha \mid \beta} \dot{x}^{\alpha} \dot{x}^{\beta}\right)=0 .
$$

Noting that $u_{\alpha, \beta} \times{ }^{\cdot \beta}=u_{\alpha}{ }_{\alpha}$, writing $f^{\mu_{\beta}}$ explicitly via the inverse of the metric (24) and using ${ }^{\alpha} f_{\mu \alpha}$ $=0$, this becomes

$\ddot{x}^{\mu}+\gamma^{\mu}{ }_{\alpha \beta} \dot{x}^{\alpha} \dot{x}^{\beta}+u_{\alpha} \dot{x}^{\alpha}\left(\bar{h}^{\mu \sigma}+u^{\mu} \bar{h}^{\sigma v} u_{v}\right) f_{\sigma \beta} \dot{x}^{\beta}-u^{\mu}\left(\dot{u}_{\alpha} \dot{x}^{\alpha}-\gamma^{\sigma}{ }_{\alpha \beta} u_{\sigma} \dot{x}^{\alpha} \dot{x}^{\beta}\right)=0$.

Now contracting this with $\mathrm{u}_{\mu}$ and using $\mathrm{u}_{\mu} \mathrm{u}^{\mu}=-1$, one obtains a constant of motion for the geodesic equation as

$$
\mathrm{u}_{\mu} \mathrm{x}^{\cdot \mu}=-\mathrm{e}=\text { constant. }
$$

Meanwhile setting the free index $\mu=i$ in (26) and using $u^{\mu}=-\delta^{\mu}$, one also finds

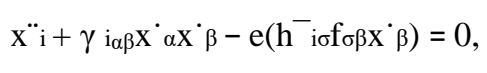

or equivalently

$$
\mathrm{x}^{\cdot \mu} \mathrm{x}^{\cdot \mathrm{i}} \mid \mu=\mathrm{eh}^{-{ }^{\mathrm{i} \sigma}} \mathrm{f}_{\sigma \mu} \mathrm{x}^{\cdot \mu},(\mathrm{i}=1,2, \ldots, \mathrm{D}-1),(28) \text { i.e. the analogous }(\mathrm{D}-1)-
$$

dimensional Lorentz force equation for a charged point particle (of charge/massratioequaltoe) writteninthecorrespondingRiemannianbackground. Moreover, contracting (28) further by $h_{\mathrm{ij}} \mathrm{x}^{\cdot \mathrm{j}}$ and using the antisymmetry of $\mathrm{f}_{\mu v}$, one obtains a second constant of motion

$$
h_{i j} \dot{x}^{i} \dot{x}^{j}=\ell^{2}>0 \text {. }
$$

Since

$$
g_{\mu \nu} \dot{x}^{\mu} \dot{x}^{\nu}=h_{i j} \dot{x}^{i} \dot{x}^{j}-\left(u_{\mu} \dot{x}^{\mu}\right)^{2}=\ell^{2}-e^{2},
$$

one concludes that the nature of the geodesics necessarily depends on the sign $\ell^{2}-e^{2}$. of 
Note in fact that the existence of these two constants of motion $e$ and follow naturally from the very structure of the metric (3). Equation (27) is a simple consequence of the fact that $\mathrm{u}^{\mu}$ is a Killing vector and tangent to a timelike geodesic curve when $\mathrm{u}_{\mathrm{k}}=$ constant, as already pointed out in section 1 . Furthermore, since $\mathrm{g}_{\mu v} \mathrm{X}^{\cdot \mu} \mathrm{X}^{\cdot v}=-1,0$ along the geodesic curve, (29) follows immediately. However one still needs (24) and (25) to obtain (28) explicitly.

Let us now take a specific example and examine the behaviour of the geodesics of this metric in more detail. Consider the simple solution discussed in section 2.1 of [4]. These odddimensional Godel-type metrics with flat backgrounds are simply characterized" by $\mathrm{u}_{\mathrm{i}}=$ $\mathrm{bJ}_{\mathrm{ij}} \mathrm{x}^{\mathrm{j}} / 2$, where $b$ is a real constant and $\mathrm{J}_{\mathrm{ij}}$ is fully antisymmetric with constant components that satisfy ${ }^{k}{ }_{j} J^{i}{ }_{k}=-\delta_{j}^{i}$. These yield $\mathrm{f}_{\mathrm{ij}}=\mathrm{bJ}_{\mathrm{ij}}, \mathrm{f}_{0 \mu}=0$ and it is readily seen that the 'Maxwell equation' $\partial_{\mathrm{i}} \mathrm{f}_{\mathrm{ij}}=0$ holds trivially. It was shown in [4] that this solution can be thought of as describing a spacetime filled with dust or as a solution to the Einstein-Maxwell theory when $\mathrm{D}=5$. However, for odd $\mathrm{D}>5$, it could as well be considered as a solution of Einstein theory coupled with a perfect fluid source with negative pressure.

Employing these specifications, the (D-1)-dimensional Lorentz force equation (28) can be put in the form

$$
\mathbf{x}^{\prime \prime}=\omega \mathbf{J}^{\cdot},
$$

where we have defined $\omega=$ eb and used a matrix notation so that $\mathbf{x}$ denotes the $(D-1) \times 1$ column vector with entries $\mathrm{x}^{\mathrm{i}}$ and $\mathbf{J}$ denotes the $(\mathrm{D}-1) \times(\mathrm{D}-1)$ matrix with components $\mathrm{J}_{\mathrm{ij}}$. Defining a second column vector $\mathbf{y}$ as $\mathbf{y}=\mathbf{x}^{\cdot}$, (30) can be integrated as

$$
\mathbf{y}=\mathrm{e} \omega \tau \mathbf{J} \mathbf{X} 0,
$$

for some constant column vector $\mathbf{x}_{0}$. Since $\mathbf{J}^{2}=-\mathbf{I}_{\mathrm{D}^{-1}}$ (the (D-1) $\times(\mathrm{D}-1)$ identity matrix and keep in mind that $D$ is odd) by construction, this can also be written as

$$
\mathbf{y}=\mathbf{x}^{\cdot}=(\cos \omega \tau+\mathbf{J} \sin \omega \tau) \mathbf{x}_{0}
$$

or integrating once again

$$
\mathbf{x}^{(\tau)}=\frac{1}{\omega}(\sin \omega \tau-\mathbf{J} \cos \omega \tau) \mathbf{x}_{0}+\mathbf{x}_{1}
$$

for a new constant column vector $\mathbf{x}_{1}$. Note that

$$
\left(\mathbf{x}-\mathbf{x}_{1}\right)^{T}\left(\mathbf{x}-\mathbf{x}_{1}\right)=\frac{1}{\omega^{2}} \mathbf{x}_{0}^{T} \mathbf{x}_{0} .
$$

Defining a scalar as $\mathcal{R}^{2} \equiv \mathbf{x}_{0}{ }^{T} \mathbf{x}_{0} / \omega^{2}$, one finds that $\left|\mathbf{x}-\mathbf{x}_{1}\right|^{2}=\mathrm{R}^{2}$, which in general is the equation of a $(D-2)$-dimensional sphere with radius $R$ and centre at $\mathbf{x}_{1}$. The equation describing $\mathrm{x}^{0}$ is given by (27), which reads 


$$
\dot{x}^{0}+\frac{b}{2} \dot{\mathbf{x}}^{T} \mathbf{J x}+e=0
$$

in matrix form. Using (31), this can be written as

$$
\dot{x}^{0}+\left(e+\frac{1}{2} b \omega \mathcal{R}^{2}\right)+\frac{b}{2}\left(b_{1} \sin \omega \tau+b_{2} \cos \omega \tau\right)=0
$$

for some constants $b_{1} \equiv \mathbf{x}_{0}{ }^{\mathrm{T}} \mathbf{x}_{1}$ and $\mathrm{b}_{2} \equiv \mathbf{x}_{0}^{\mathrm{T}} \mathbf{J} \mathbf{x}_{1}$. Integrating this equation, one finally finds

$$
x^{0}(\tau)=-e\left(1+\frac{1}{2} b^{2} \mathcal{R}^{2}\right) \tau+\frac{1}{2 e}\left(b_{1} \cos \omega \tau-b_{2} \sin \omega \tau\right)+b_{3}
$$

for a new integration constant $b_{3}$. Note that the case for the null geodesics corresponds simply to taking $b R=1$. Since the coefficient of the term linear in $\tau$ is never zero, $x^{0}(\tau)$ cannot be periodic. Hence we conclude that the geodesic curves move on $\mathrm{R} \times \mathrm{S}^{\mathrm{D}-2}$ and are complete for this special solution in odd dimensions.

Note, in fact, that this result can also be generalized to the case when the rank of $\mathbf{J}$ is $2 \mathrm{k}$ where $22 k \leqslant D-1$ and $\mathbf{J}^{2}=-\mathbf{I}_{2 \mathrm{k}}$, the $2 \mathrm{k} \times 2 \mathrm{k}$ identity matrix. Then $2 \mathrm{k}$ of the spatial coordinates will obey the Lorentz force equation

$$
\mathrm{x}^{\cdot \cdot \mathrm{i}}=\omega \mathrm{J}_{\mathrm{ij}} \mathrm{x}^{\cdot \mathrm{j}}, \quad(\mathrm{i}=1,2, \ldots, 2 \mathrm{k}),
$$

whereas the remaining spatial ones will simply satisfy

$$
\mathrm{x}^{-\cdot \mathrm{i}}=0, \quad(\mathrm{i}=2 \mathrm{k}+1, \ldots, \mathrm{D}-1) .
$$

Thereforethe $2 \mathrm{k}$-dimensionalpartwillbeperiodicin $\tau$ andwilldescribea(2k-1)-dimensional sphere whereas the remaining parts will be linear in $\tau$. Hence the geodesic curves will move on $\mathrm{R}^{\mathrm{D}-2 \mathrm{k}} \times \mathrm{S}^{2 \mathrm{k}-1}$ in this case.

As yet another example, one can also consider the geodesics of the spacetime described by (6). If one uses Cartesian coordinates $(\mathrm{x}, \mathrm{y}, \mathrm{z})$ instead of the circular cylindrical coordinates used in (6), the geodesics can alternatively be found by extremizing the Lagrangian

$$
\mathrm{L}=\mathrm{x}^{\cdot 2}+\mathrm{y}^{\cdot 2}+\mathrm{z}^{\cdot 2}-\left(\mathrm{t}^{\cdot}+\mathrm{s}(\mathrm{x}(\tau), \mathrm{y}(\tau)) \mathrm{z}\right)^{\cdot 2}=\lambda,
$$

where $\mathrm{s}(\mathrm{x}, \mathrm{y})$ isaharmonic function ofitsarguments, adot denotes differentiation withrespect to the affine parameter $\tau$ and $\lambda=-1,0$ for timelike and null geodesics, respectively. One easily obtains two constants of motion from the $t$ and $\mathrm{z}$ variations as

$\mathrm{t}^{\cdot}+\mathrm{s}(\mathrm{x}(\tau), \mathrm{y}(\tau)) \mathrm{z}^{\cdot}=-\mathrm{e}=$ constant $\quad$ and $\quad \dot{z}+e s(x(\tau), y(\tau))=\ell=$ constant,

whereas the $x$ and $y$ variations yield

$\ddot{x}-e(\partial s / \partial x)(\ell-e s(x, y))=0 \quad$ and $\quad \ddot{y}-e(\partial s / \partial y)(\ell-e s(x, y))=0$, respectively. Note that using the constants of motion in the expression (33) for the Lagrangian, one now also has the constraint

$$
\dot{x}^{2}+\dot{y}^{2}=\lambda+e^{2}-(\ell-e s(x(\tau), y(\tau)))^{2} .
$$


In general, this system of coupled ordinary differential equations can be solved given the explicit form of the harmonic function $\mathrm{s}(\mathrm{x}, \mathrm{y})$.

Let us now consider as a simple example, the harmonic function

$$
\mathrm{s}(\mathrm{x}, \mathrm{y})=\gamma+\alpha \mathrm{x}+\beta \mathrm{y},
$$

corresponding to the $\mathrm{n}=1$ case in (13). Keeping in mind that we are after closed timelike or null geodesics, one can now easily integrate (35), set the coefficient of the linear term in $\tau$ to zero and obtain the periodic functions $\mathrm{x}(\tau)$ and $\mathrm{y}(\tau)$. Using these in (34), one then calculates $\mathrm{z}(\tau)$ (which itself turns out to be a periodic function in $\tau$ ) and $\mathrm{t}(\tau)$ as

$$
t(\tau)=-\frac{e}{2}\left(1-\frac{\lambda}{e^{2}}\right) \tau+g(\tau)
$$

where the constraint (36) has been used and $g(\tau)$ contains all the parts periodic in $\tau$. Hence for closed geodesics, one needs that $\lambda=\mathrm{e}^{2}$ ! However, this is not possible ${ }^{1}$ and one concludes that there are no closed timelike or null geodesics in the spacetime described by $(6)$ when $\mathrm{s}(\mathrm{x}, \mathrm{y})$ is given by (37). We conjecture that this result can also be generalized to the case of the more general harmonic function $\mathrm{s}(\mathrm{x}, \mathrm{y})$ given by (13).

\section{Conclusions}

In this work, it has been verified explicitly that the spacetimes described by Godel-type metrics" with both flat and non-flat backgrounds always have closed timelike or null curves, provided that at least one of the $u_{i}\left(x^{\ell}\right) \neq$ constant. It has also been shown that the geodesics of Godel-type metrics with constant" $\mathrm{u}_{\mathrm{k}}$ are characterized by the (D - 1)-dimensional Lorentz force equation for a charged point particle formulated in the corresponding Riemannian background. A specific example in odd dimensions has been considered for which timelike and null geodesics are complete and never closed. It has also been laid out in a separate example that there are no closed timelike or null geodesics in the spacetime described by (6) when $\mathrm{s}(\mathrm{x}, \mathrm{y})$ is a linear function of its arguments.

One possible direction to look at would be to examine the existence of closed timelike curves in spacetimes described by the most general Godel-type metrics with non-flat" backgrounds and non-constant $u_{k}$.

\section{Acknowledgments}

RJG is supported by CONICET (Argentina) and in part by a grant from the National University of Cordoba (Argentina) and by grant NSF-INT-0204937 of the National Science Foundation of theUSA. MG,AK and OSarepartiallysupported bytheScientific and

\footnotetext{
${ }^{1}$ Note that when $\mathrm{e}=0,(35)$ and (34) are easily solved yielding $\mathrm{x}(\tau), \mathrm{y}(\tau)$ and $\mathrm{z}(\tau)$ as linear functions in $\tau$ and $\mathrm{t}(\tau)$ as a quadratic function in $\tau$, which obviously do not describe closed geodesics then.
} 
Technological Research" Council of Turkey (TUB" 'ITAK). MG is also supported in part by the Turkish Academy of Sciences (TUBA)."

\section{References}

[1] Godel K 1949“* Rev. Mod. Phys. 21 447-50

[2] Stephani H, Kramer D, MacCallum M A H, Hoenselaers C and Herlt E 2002 Exact Solutions of Einstein's Field Equations 2nd edn (Cambridge: Cambridge University Press)

[3] Hawking S and Ellis G F R 1977 The Large Scale Structure of Space-Time (Cambridge: Cambridge University Press)

[4] Gurses M, Karasu A and Sarı” glu $\quad$ O 2005“ Class. Quantum Grav. 22 1527-43 (Preprint hepth/0312290)

[5] Gurses M and Sarı* glu ${ }^{-} \quad$ O 2005* Class. Quantum Grav. 22 4699-713 (Preprint hepth/0505268)

[6] Boyda E K, Ganguli S, Ho`rava P and Varadarajan U 2003 Phys. Rev. D 67106003 (Preprint hep-th/0212087)

[7] Brecher D, DeBoer P A, Rozali M and Page D C 2003 J. High Energy Phys. JHEP10(2003)031 (Preprint hep-th/0306190)

[8] Rebouc, as M J and Tiomno J 1983 Phys. Rev. D 28 1251-64

[9] Barrow J D and Dabrowski M P 1998 Phys. Rev. D 58103502 (Preprint hep-th/9803048)

[10] Accioly A J 1987 Nuovo Cimento B 100 703-7

[11] Clifton T and Barrow J D 2005 Phys. Rev. D 72123003 (Preprint gr-qc/0511076) 